\title{
Determinacy of Equilibrium in Outcome Game Forms
}

\author{
by
}

\author{
Cristian Litan, \\ Francisco Marhuenda \\ and \\ Peter Sudhölter
}

Discussion Papers on Business and Economics

No. $17 / 2014$

FURTHER INFORMATION

Department of Business and Economics Faculty of Business and Social Sciences University of Southern Denmark

Campusvej 55

DK-5230 Odense M

Denmark

Tel.: +456550 3271

Fax: +456550 3237

E-mail: lho@sam.sdu.dk

http://www.sdu.dk/ivoe 


\title{
Determinacy of Equilibrium in Outcome Game Forms*
}

\author{
Cristian Litan ${ }^{\dagger} \quad$ Francisco Marhuenda ${ }^{\ddagger} \quad$ Peter Sudhölter $^{\S}$
}

November 4, 2014

\begin{abstract}
We show the generic finiteness of the number of probability distributions on outcomes induced by Nash equilibria for two-person game forms such that either (i) one of the players has no more than two strategies or (ii) both of the players have three strategies, and (iii) for outcome game forms with three players, each with at most two strategies. Finally, we exhibit an example of a game form with three outcomes and three players for which the Nash equilibria of the associated game induce a continuum of payoffs for an open non-empty set of utility profiles.
\end{abstract}

Keywords: Outcome game form · Completely mixed Nash equilibrium · Generic finiteness

JEL Classification: C72

\section{Introduction}

In normal form games with an arbitrary number of players the payoffs of which may be perturbed independently Rosenmüller (1971) and Wilson (1971) simultaneously proved (see also Harsanyi 1973) that generically there is a finite number of equilibria. This result was extended to extensive form games by Kreps and Wilson (1982). On the other hand, Govindan and McLennan (2001) and Kukushkin, Litan, and Marhuenda (2008) show that the situation for outcome game forms is entirely different. These authors construct some game forms for which there is a continuum of probability distributions on outcomes induced by the Nash equilibria of the associated games for an open non-empty set of utility profiles.

A natural question that arises is to try to determine which outcome game forms admit a finite number of probability distributions on outcomes induced by Nash equilibria. For example, Mas-Colell (2010) proved that for two player game forms the equilibrium payoffs are generically finite, and Govindan and McLennan (2001) proved that for game forms with two outcomes and any number of players the number of equilibrium distributions is generically finite. Similar results are obtained for game forms with two players and three outcomes (González-Pimienta 2010) and sender-receiver cheap-talk games (Park 1997). Using semi-algebraic geometry techniques Govindan and McLennan (1998) showed in an unpublished manuscript the generic finiteness of the number of equilibrium distributions on outcomes, when the associated game is either a two player zero sum or a common interest game. This result is also proved by Litan and Marhuenda (2012) using elementary linear algebra.

${ }^{*}$ Cristian Litan acknowledges financial support by the University Babeş-Bolyai under the project GTC-34068/2013. Francisco Marhuenda acknowledges financial support by the Spanish Ministerio de Ciencia e Innovación under project ECO2013-42710-P. Peter Sudhölter was supported by The Danish Council for Independent Research|Social Sciences under the FINQ project and by the Spanish Ministerio de Ciencia e Innovación under project ECO2012-33618.

${ }^{\dagger}$ Department of Statistics, Forecasting, Mathematics, Faculty of Economics and Business Administration, Babeş-Bolyai University, Cluj Napoca, Romania. E-mail: cristian.litan@econ.ubbcluj.ro

${ }_{\ddagger}^{\ddagger}$ Department of Economics, University Carlos III of Madrid, Calle Madrid 126, 28903-Getafe (Madrid), Spain. E-Mail: marhuend@eco.uc3m.es

$\S$ Department of Business and Economics and COHERE, University of Southern Denmark, Campusvej 55, 5230 Odense M, Denmark. E-mail: psu@sam.sdu.dk 
In this paper we prove the generic finiteness of the number of probability distributions on outcomes induced by Nash equilibria for two-person outcome game forms in which one of the players has no more than two strategies or both of the players have three strategies. The results of González-Pimienta (2010) and Govindan and McLennan (2001) imply that for two-person outcome game forms with at most three outcomes the number of equilibrium distributions is generically finite. We provide an example of an outcome game form with three outcomes and three players for which the Nash equilibria of the associated games induce a continuum of probability distributions on outcomes for an open non-empty set of utility profiles. Finally, we show that for outcome game forms with three players, each with at most two strategies, generic finiteness of the number of probability distributions on outcomes induced by Nash equilibria is obtained.

\section{Outcome game forms}

A (finite, pure) L-person outcome game form (on $\Omega$ ) is defined by Govindan and McLennan (2001) as a tuple $\left(S^{1}, \ldots, S^{L}, \phi\right)$ such that, for all $i \in\{1, \ldots, L\}, S^{i}$ is a finite nonempty set, and $\phi: S \rightarrow \Omega$, where $\Omega$ is a finite nonempty set (the set of outcomes) and $S=S^{1} \times \cdots \times S^{L}$. A profile $u=\left(u^{1}, \ldots, u^{L}\right) \in$ $\mathbb{R}^{\Omega} \times \cdots \times \mathbb{R}^{\Omega}$ defines the associated finite $L$-person game $g_{u}^{\phi}=\left(S^{1}, \ldots, S^{L}, u^{1} \circ \phi, \ldots, u^{L} \circ \phi\right)$, where "०" denotes "composition". Recall that a Nash equilibrium (NE) $\left(x^{1}, \ldots, x^{L}\right)$ of $g_{u}^{\phi}$ is a completely mixed $\mathrm{NE}$ (CMNE) if $x^{i} \in \Delta_{+}\left(S^{i}\right)$ for all $i=1, \ldots, L$, where $\Delta_{+}\left(S^{i}\right)$ denotes the set of strictly positive probability measures on $S^{i}$.

We say that a subset of a Euclidean space is generic if it contains an open and dense subset of this Euclidean space.

\section{Distributions on outcomes and minimality for two-person out- come game forms}

Let $\left(S^{1}, S^{2}, \phi\right)$ be an outcome game form with two players, where $S^{1}=\{1,2, \ldots, m\}, S^{2}=\{1,2, \ldots, n\}$ and $S=S^{1} \times S^{2}$. For each outcome $\omega \in \Omega$ the mapping $\phi$ defines the $m \times n$ matrix $\phi^{\omega}$ with values in $\{0,1\}$, whose $(r, s)$ entry is 1 if $\phi(r, s)=\omega$ and 0 otherwise. For $r \in S^{1}, s \in S^{2}$ denote $\phi_{r s}=\phi(r, s) \in \Omega$. To each $u \in \mathbb{R}^{\Omega}$ we assign the matrix

$$
M(u)=\sum_{\omega \in \Omega} u(\omega) \phi^{\omega}
$$

Let $u=\left(u^{1}, u^{2}\right) \in \mathbb{R}^{\Omega} \times \mathbb{R}^{\Omega}$. A pair of strategies $(x, y) \in \Delta\left(S^{1}\right) \times \Delta\left(S^{2}\right)$ is a Nash equilibrium (NE) if $x M\left(u^{1}\right) y \geqslant M_{i} \cdot\left(u^{1}\right) y$ and $x M\left(u^{2}\right) y \geqslant x M_{\cdot j}\left(u^{2}\right)$ for all $i \in S^{1}$ and $j \in S^{2}$, where throughout, for $u \in \mathbb{R}^{\Omega}$, $M_{i .}(u)$ and $M_{\cdot j}(u)$ denote row $i$ and column $j$ of the matrix $M(u), x M(u)$ and $M(u) y$ are regarded as elements of $\mathbb{R}^{n}$ and $\mathbb{R}^{m}$, and the scalar product of $z$ and $z^{\prime}, z, z^{\prime} \in \mathbb{R}^{k}$, is written $z \cdot z^{\prime}$ or simply as $z z^{\prime}$. The strategies $x \in \Delta\left(S^{1}\right)$ and $y \in \Delta\left(S^{2}\right)$ of the players induce a probability distribution on $\Omega$ that assigns the probability $x \phi^{\omega} y$ to the outcome $\omega \in \Omega$.

Let $\theta=\left(\theta_{\omega}\right)_{\omega \in \Omega}$ be the vector of $|\Omega|$ variables. Then, $M[\theta]=\sum_{\omega \in \Omega} \theta_{\omega} \phi^{\omega}$ is a matrix the $r s$ entry $\theta_{r s}$ of which is $\theta_{\phi(r, s)}$. Note that the matrix $M[\theta]$ determines the outcome game form $\phi$ in a trivial way by setting $\phi(r, s)=\theta_{r s}$ Hence, we identify the matrix $M[\theta]$ with the outcome game form $\left(S^{1}, S^{2}, \phi\right)$.

Let

$$
\mathcal{V}=\left\{u \in \mathbb{R}^{\Omega}:|\{u(w): w \in \Omega\}|=|\Omega|\right\} .
$$

Then $\mathcal{V}$ is a generic subset of $\mathbb{R}^{\Omega}$.

Kukushkin, Litan, and Marhuenda (2008) provide an example of an outcome game form with two players in which, for a non-empty open set of utility profiles, there is a continuum of outcome distributions induced by the Nash equilibria. In that example the first player has three strategies and the second player has four strategies. The next theorem shows the example is minimal in terms of strategies. 
Theorem 3.1. If $\left(S^{1}, S^{2}, \phi\right)$ is a 2-person outcome game form such that

$$
\min \left\{\left|S^{1}\right|,\left|S^{2}\right|\right\} \leqslant 2 \text { or }\left|S^{1}\right|=\left|S^{2}\right|=3,
$$

then there is a generic set $\mathcal{W} \subseteq \mathbb{R}^{\Omega}$ such that for any $u^{1}, u^{2} \in \mathcal{W}$ the set of CMNEs of the game $g_{u}^{\phi}$ induce finitely many probability distributions on outcomes.

Proof. We may assume that the rows of $\phi$ (that is, $\phi_{r} .=\left(\phi_{r s}\right)_{s \in S^{2}}, r \in S^{1}$ ) are pairwise distinct. Indeed, for any utility profile in $\mathbb{R}^{\Omega} \times \mathbb{R}^{\Omega}$ the set of distributions on outcomes induced by (completely mixed) Nash equilibria is not changed if multiple rows are eliminated. A similar assumption refers to the columns of $\phi$ (that is, $\left.\phi_{\cdot s}=\left(\phi_{r s}\right)_{r \in S^{1}}, s \in S^{2}\right)$. Hence, we may assume without loss of generality that

$$
\left|\left\{\phi_{r .}: r \in S^{1}\right\}\right|=m, \quad\left|\left\{\phi_{\cdot s}: s \in S^{2}\right\}\right|=n, \quad \text { and } \quad m \leqslant n .
$$

Let $\left(u^{1}, u^{2}\right) \in \mathcal{V} \times \mathcal{V}$. For $(r, s) \in S^{1} \times S^{2}$ denote $u_{r s}=u^{2}\left(\phi_{r s}\right)$. We distinguish three cases.

Case 1: $m=1$

As $u^{2} \in \mathcal{V}$, any Nash equilibrium selects the unique $\arg \max _{s_{2} \in S_{2}} u^{2}\left(\phi_{1 s}\right)$, and, hence a CMNE can only exist if $n=1$.

Case 2: $m=2$

If there are $s, s^{\prime} \in S^{2}, s \neq s^{\prime}$, such that $\phi_{1 s}=\phi_{1 s^{\prime}}$, then let $(x, y)$ be a Nash equilibrium. If $u^{2} \in \mathcal{V}$, then by (1) we may assume that $u^{2}\left(\phi_{2 s}\right)<u^{2}\left(\phi_{2 s^{\prime}}\right)$ so that $y_{s}=0$. Thus, $(x, y)$ is not completely mixed and there is nothing to prove in this subcase. Similarly, we may proceed if $\phi_{2 s}=\phi_{2 s^{\prime}}$. Therefore, we shall now assume that

$$
n=\left|\left\{\phi_{r s}: s \in S^{2}\right\}\right| \text { for } r=1,2 .
$$

If $n=2$, let $\mathcal{W}=\{u \in \mathcal{V}: h(u) \neq 0\}$, where $h(\theta)=\theta_{11} \theta_{22}-\theta_{12} \theta_{21}$ is a polynomial in $|\Omega|$ variables. Notice that $h \neq 0$, by (1) and (2). Thus, the set $\mathcal{W}$ is generic and there exists at most one completely mixed Nash equilibrium provided that $u^{1}, u^{2} \in \mathcal{W}$.

We now assume that $n \geqslant 3$. Define the polynomial $f$ in $|\Omega|$ variables by

$$
f(\theta)=\left(\theta_{11}-\theta_{12}\right)\left(\theta_{21}-\theta_{23}\right)-\left(\theta_{11}-\theta_{13}\right)\left(\theta_{21}-\theta_{22}\right) .
$$

Claim: If $f\left(u^{2}\right) \neq 0$ then the game $g_{u}^{\phi}$ has no CMNE.

If $x$ is a mixed strategy of 1 such that 2 is indifferent between the payoff columns $u_{\cdot 1}, u_{\cdot 2}$, and $u_{\cdot 3}$, then

$$
x \cdot\left(\begin{array}{lll}
u_{11}-u_{12} & u_{11}-u_{13} & 1 \\
u_{21}-u_{22} & u_{21}-u_{23} & 1
\end{array}\right)=(0,0,1) .
$$

We conclude that

$$
\operatorname{det}\left(\begin{array}{ccc}
u_{11}-u_{12} & u_{11}-u_{13} & 1 \\
u_{21}-u_{22} & u_{21}-u_{23} & 1 \\
0 & 0 & 1
\end{array}\right)=0
$$

Therefore $f\left(u^{2}\right)=0$ so that our claim has been proved.

Now the proof in this case can be completed. By (1) there exists $s \in S^{2}$ such that $\phi_{1 s} \neq \phi_{2 s}$. By (2) there exists $s^{\prime} \in S^{2} \backslash\{s\}$ such that $\phi_{2 s^{\prime}} \neq \phi_{1 s}$ so that we may assume without loss of generality that

$$
\phi_{11} \neq \phi_{21} \text { and } \phi_{11} \neq \phi_{22} \text {. }
$$

By (2) and (5),

$$
\phi_{11} \notin\left\{\phi_{12}, \phi_{13}, \phi_{21}, \phi_{22}\right\} .
$$


Let $\omega=\phi_{11}, \omega \in \Omega$. Let $u \in \mathcal{V}$ such that $f(u)=0$. For $\varepsilon>0$ let $u^{\varepsilon} \in \mathbb{R}^{\Omega}$ differ from $u$ only in as much as $u^{\varepsilon}(\omega)=u(\omega)+\varepsilon$. If $\phi_{23} \neq \omega$, then $f\left(u^{\varepsilon}\right)=f(u)+\varepsilon\left(u_{22}^{\varepsilon}-u_{23}^{\varepsilon}\right)=\varepsilon\left(u_{22}-u_{23}\right) \neq 0$. If $\phi_{23}=\omega$, then

$$
f\left(u^{\varepsilon}\right)=\varepsilon\left(u_{22}+u_{12}-2 u_{11}\right)-\varepsilon^{2} .
$$

Hence, we conclude that $f \neq 0$, so that $\mathcal{W}=\{u \in \mathcal{V}: f(u) \neq 0\}$ is generic. By our claim, $\mathcal{W}$ has the desired properties.

Case 3: $m=n=3$

It suffices to describe a suitable generic set of utilities for player 2 . We distinguish 2 cases.

(1) There exists $i \in S_{1}$ such that $\phi_{i 1}=\phi_{i 2}=\phi_{i 3}$. We may assume that $i=3$. Let $u \in \mathcal{V}$. If $x^{\prime}$ is a completely mixed strategy of 1 such that 2 is indifferent between the columns, then let $x=\frac{x_{\{1,2\}}^{\prime}}{x_{1}+x_{2}} \in \Delta_{+}(\{1,2\})$ and observe that (4) must hold. Also, we may assume that (2) holds, because otherwise there exist two payoff columns that differ only in one coordinate so that a completely mixed Nash equilibrium is ruled out. By (1) there exists $\ell \in S_{2}$ such that $\phi_{1 \ell} \neq \phi_{2 \ell}$ so that (5) may be assumed and the proof may be finished by literally copying the corresponding part of the case $m=2$.

(2) For any $k \in S_{1},\left|\left\{\phi_{k \ell}: \ell \in S_{2}\right\}\right| \geqslant 2$.

Consider again the polynomial $f$ defined in (3). If $x$ is a mixed strategy that makes 2 indifferent between all columns, then

$$
x \cdot\left(\begin{array}{lll}
u_{11}-u_{12} & u_{11}-u_{13} & 1 \\
u_{21}-u_{22} & u_{21}-u_{23} & 1 \\
u_{31}-u_{32} & u_{31}-u_{33} & 1
\end{array}\right)=(0,0,1) .
$$

Claim: If the system of equations (7) has multiple solutions, then $f\left(u^{2}\right)=0$ and $\left|\left\{\phi_{k \ell}: \ell \in S_{2}\right\}\right|=$ 3 , for every $k \in S_{1}$.

As (7) has multiple solutions, the columns of the matrix are not linearly independent. Thus, there exists $z \in \mathbb{R}^{3}, z \neq 0$, such that

$$
\left(\begin{array}{ccc}
u_{11}-u_{12} & u_{11}-u_{13} & 1 \\
u_{21}-u_{22} & u_{21}-u_{23} & 1 \\
u_{31}-u_{32} & u_{31}-u_{33} & 1
\end{array}\right) \cdot z=(0,0,0)
$$

By (7) we conclude that $z_{3}=0$. Moreover, as $u \in \mathcal{V}$, our assumptions imply that $z_{1} \neq 0$ and, similarly $z_{2} \neq 0$. Hence, we may assume (after replacing $z$ by $z / z_{1}$ if necessary) that $z_{1}=1$. Hence, with $\lambda=-z_{2}$, we have

$$
\begin{aligned}
& u_{11}-u_{12}=\lambda\left(u_{11}-u_{13}\right) \\
& u_{21}-u_{22}=\lambda\left(u_{21}-u_{23}\right) \text { and } \\
& u_{31}-u_{32}=\lambda\left(u_{31}-u_{33}\right) .
\end{aligned}
$$

Thus, $f\left(u^{2}\right)=0$. As by our assumption, one of these differences in each row is nonzero, all of them are nonzero and we conclude that $\left|\left\{\phi_{k \ell}: \ell \in S_{2}\right\}\right|=3$ for all $k \in S_{1}$ and the claim follows.

Now the proof can be completed. If, for any $u \in \mathcal{V},(7)$ has one or no solution, then $\mathcal{W}=\mathcal{V}$ has the desired properties. In the other case, let $u \in \mathcal{V}$ such that (7) has multiple solutions. Hence, $\left|\left\{\phi_{k \ell}: \ell \in S_{2}\right\}\right|=3$ for all $k \in S_{1}$. By (1) we conclude that $\left\{\phi_{1 \ell}: \ell=1,2,3\right\} \neq\left\{\phi_{2 \ell}: \ell=1,2,3\right\}$ so that we may assume that $\phi_{11} \neq \phi_{2 \ell}, \ell=1,2,3$. Let $\varepsilon>0$ be small enough such that $\widetilde{u} \in \mathcal{U}$, where $\widetilde{u}$ is the utility function that differs from $u$ only inasmuch as $\widetilde{u}\left(\phi_{11}\right)=u\left(\phi_{11}\right)+\varepsilon$. Then $f(\widetilde{u})=f(u)+\varepsilon\left(u_{22}-u_{23}\right) \neq 0$ so that $f$ is not the zero polynomial. Thus, $\mathcal{W}=\{u \in \mathcal{V}: f(u) \neq 0\}$ is a generic set with the desired properties. 


\section{Outcome game forms with three players}

The first example of an outcome game form in which there is a continuum of distributions on outcomes induced by the Nash equilibria of the associated games for an open non-empty set of utility profiles was provided by Govindan and McLennan (2001). Their example had three players and six outcomes. On the other hand, for any outcome game form with two outcomes they prove generic finiteness of the number of Nash equilibrium outcome distributions, and González-Pimienta (2010) shows this generic finiteness for two-person game forms with three outcomes. Finally, Kukushkin, Litan, and Marhuenda (2008) provide an example of an outcome game form with two players and four outcomes in which there is a continuum of outcome distributions induced by the Nash equilibria of the associated games for an open non-empty set of utility profiles. Thus, it is natural to ask if four is the minimum number of outcomes needed to construct outcome game forms which do not generically have finitely many distributions on $\Omega$ induced by Nash equilibria. However, the next example shows that the results in González-Pimienta (2010) cannot be extended to three players. That is, a three-person game form with three outcomes $a, b$, and $c$ may allow a continuum of outcome distributions induced by Nash equilibria for an open non-empty set of utility profiles.

In Section 4.2 we show the generic finiteness for outcome game forms with three players, each with at most two strategies.

\subsection{An example with three players and three outcomes}

Let $\Omega=\{a, b, c\}, S_{1}=\{N, E, S, W\}, S_{2}=\{L, R\}$ and $S_{3}=\{U, D\}$. We use the notation of Section 3 of Govindan and McLennan (2001) and consider the game form

$$
\begin{aligned}
& \text { U D } \\
& L R \quad L R \\
& \begin{array}{c}
N \\
E \\
S \\
W
\end{array} \quad\left(\begin{array}{ll}
a & a \\
b & b \\
a & c \\
b & a
\end{array}\right) \quad \begin{array}{l}
N \\
E \\
W
\end{array} \quad\left(\begin{array}{cc}
c & c \\
a & a \\
a & c \\
b & a
\end{array}\right)
\end{aligned}
$$

so that player 1 selects the row, player 2 the column, and player 3 the matrix. Moreover, for $i \in\{1,2,3\}$, $a_{i}=u_{i}(a), b_{i}=u_{i}(b)$, and $c_{i}=u_{i}(c)$. If $a_{i}>\max \left\{b_{i}, c_{i}\right\}$ for all $i \in\{1,2,3\}$, then we may define, for any $p$ with $0 \leqslant p \leqslant t:=\frac{a_{3}-b_{3}}{2 a_{3}-b_{3}-c_{3}}$, the strategy profile $X(p)=((p, q, r, s),(y, 1-y),(z, 1-z))$ by

$$
\begin{aligned}
z & =\frac{a_{1}-c_{1}}{2 a_{1}-b_{1}-c_{1}}=y \\
q & =\frac{a_{3}-c_{3}}{a_{3}-b_{3}} p \\
r & =\left(1-\left(\frac{2 a_{3}-b_{3}-c_{3}}{a_{3}-b_{3}}\right) p\right) \frac{a_{2}-b_{2}}{2 a_{2}-b_{2}-c_{2}} \\
s & =\frac{a_{2}-c_{2}}{a_{2}-b_{2}} r
\end{aligned}
$$

It is straightforward to show that $X(p)$ is a Nash equilibrium that induces the payoff $\frac{a_{1}^{2}-b_{1} c_{1}}{2 a_{1}-b_{1}-c_{1}}$ for the row player. Let $\pi_{2}(p)$ denote the payoff of the column player. We may easily compute

$$
\pi_{2}(0)=\frac{a_{2}^{2}-b_{2} c_{2}}{2 a_{2}-b_{2}-c_{2}}
$$

and

$$
\pi_{2}(t)=\frac{\left(a_{3}-b_{3}\right)\left(\left(a_{1}-c_{1}\right) a_{2}+\left(a_{1}-b_{1}\right) c_{2}\right)+\left(a_{3}-c_{3}\right)\left(\left(a_{1}-c_{1}\right) b_{2}+\left(a_{1}-b_{1}\right) a_{2}\right)}{\left(2 a_{3}-b_{3}-c_{3}\right)\left(2 a_{1}-b_{1}-c_{1}\right)}
$$


so that $\pi_{2}(0) \neq \pi_{2}(t)$ for a nonempty open subset of utility profiles. Moreover, if $\pi_{2}(0) \neq \pi_{2}(t)$, then by continuity of $\pi_{2}:[0, t] \rightarrow \mathbb{R}$, there is a continuum of payoffs of the column player induced by the completely mixed Nash equilibria $X(p), 0<p<t$. A similar statement is valid for the matrix player.

On the other hand, we show in Section 4.2 that if each of the players has at most two strategies, generically the number of probability distributions on outcomes induced by the Nash equilibria of the game is finite.

\subsection{Outcome game forms with three players and two strategies each}

Let $\Omega$ be the finite non-empty set of outcomes. We consider an outcome game form $\left(S^{1}, S^{2}, S^{3}, \phi\right)$ with three players and two strategies each, i.e., we assume that $S^{1}=S^{2}=S^{3}=\{1,2\}$. Let $\left(\theta_{\omega}\right)_{\omega \in \Omega}$ be the vector of $|\Omega|$ variables and $\left(\theta_{\omega}^{\ell}\right)_{(\omega \in \Omega, \ell \in\{1,2,3\})}$ be the vector of $3|\Omega|$ variables. We denote by $\theta_{i j k}=\theta_{\phi(i, j, k)}, \theta_{i j k}^{\ell}=\theta_{\phi(i, j, k)}^{\ell}$. Let $\mathbb{R}\left[\left(\theta_{\omega}\right)_{\omega \in \Omega}\right]$ denote the ring of real polynomials in the $|\Omega|$ variables and let $\mathbb{R}\left[\left(\theta_{\omega}^{\ell}\right)_{\omega \in \Omega, \ell \in\{1,2,3\}}\right]$ denote the ring of real polynomials in $3|\Omega|$ variables.

For any finite $\mathcal{F} \subseteq \mathbb{R}\left[\left(\theta_{\omega}^{\ell}\right)_{\omega \in \Omega, \ell \in\{1,2,3\}}\right]$, let $U_{\mathcal{F}}$ be defined by

$$
U_{\mathcal{F}}=\left\{u \in \mathbb{R}^{\Omega} \times \mathbb{R}^{\Omega} \times \mathbb{R}^{\Omega}: f(u) \neq 0 \forall f \in \mathcal{F} \backslash\{0\}\right\} .
$$

Note that $U_{\mathcal{F}}$ is an open and dense subset of $\left(\mathbb{R}^{\Omega}\right)^{3}$.

Now we are able to prove the following main result of this section.

Theorem 4.1. For any three-person pure outcome game form $\left(S^{1}, S^{2}, S^{3}, \phi\right)$ with $S^{1}=S^{2}=S^{3}=\{1,2\}$ there is a generic set $U$ of utility profiles such that, for any $u=\left(u^{1}, u^{2}, u^{3}\right) \in U$, the set of CMNEs of the game $g_{u}^{\phi}$ induce finitely many probability distributions on outcomes.

Proof. We may assume that the number of CMNEs is not generically finite. Also, by Theorem 3.1, we may assume that none of the players $i=1,2,3$ is a dummy, where $i$ is dummy if $\phi$ is invariant under any permutation of $S^{i}$. Hence, by renaming the players if necessary, we assume that, for each utility profile $u=\left(u^{1}, u^{2}, u^{3}\right)$ in an open and nonempty subset $\mathcal{Y}$ of $\mathbb{R}^{\Omega} \times \mathbb{R}^{\Omega} \times \mathbb{R}^{\Omega}$, there are infinitely many completely mixed strategies for player 1 that may be extended to CMNEs by suitably chosen strategies of the remaining players.

It suffices to show that there exists a finite $\mathcal{F} \subseteq \mathbb{R}\left[\left(\theta_{\omega}^{\ell}\right)_{\omega \in \Omega, \ell \in\{1,2,3\}}\right]$ such that, for any $u \in U_{\mathcal{F}} \cap \mathcal{Y}$, the set of probability measures on $\Omega$ induced by CMNEs is finite.

Let $u=\left(u^{1}, u^{2}, u^{3}\right) \in \mathbb{R}^{\Omega} \times \mathbb{R}^{\Omega} \times \mathbb{R}^{\Omega}$ and let $\left(\left(x_{1}, x_{2}\right),\left(y_{1}, y_{2}\right),\left(z_{1}, z_{2}\right)\right)$ be a CMNE. Define the polynomial $H \in \mathbb{R}\left[\left(\theta_{\omega}\right)_{\omega \in \Omega}\right]$ by

$$
H=\sum_{i, j, k=1}^{2} \theta_{i j k} x_{i} y_{j} z_{k} .
$$

The probability of each outcome is its coefficient in the polynomial $H$. Let $x=x_{1}, x_{2}=1-x, y=$ $y_{1}, y_{2}=1-y, z=z_{1}, z_{2}=1-z$. The polynomial $H$ may be written as

$$
H=A x y z+B x y+C x z+D y z-E x-F y-G z+\theta_{222} .
$$

where $A, \ldots, G \in \mathbb{R}\left[\left(\theta_{\omega}\right)_{\omega \in \Omega}\right]$ are defined by

$$
\begin{aligned}
& A=\theta_{111}-\theta_{121}-\theta_{112}+\theta_{122}-\theta_{211}+\theta_{221}+\theta_{212}-\theta_{222} \\
& B=\theta_{112}-\theta_{122}-\theta_{212}+\theta_{222} \\
& C=\theta_{121}-\theta_{122}-\theta_{221}+\theta_{222} \\
& D=\theta_{211}-\theta_{212}-\theta_{221}+\theta_{222} \\
& E=\theta_{222}-\theta_{122} \\
& F=\theta_{222}-\theta_{212} \\
& G=\theta_{222}-\theta_{221}
\end{aligned}
$$


Note that $A, \ldots, G$ are polynomials in $\mathbb{R}\left[\left(\theta_{\omega}\right)_{\omega \in \Omega}\right]$ with coefficients $x(u), y(u), z(u)$ which depend on $u^{1}, u^{2}, u^{3}$. For $i \in\{1,2,3\}$, each of the polynomials $A, \ldots, G$ may be identified with a polynomial $A_{i}$ in $\mathbb{R}\left[\left(\theta_{\omega}^{i}\right)_{\omega \in \Omega}\right]$ by formally replacing the variable $\theta_{\omega}$ with the variable $\theta_{\omega}^{i}$. Note that with this identification, $A_{i} \neq A_{j}$ for $i \neq j$, since $A_{i}$ and $A_{j}$ are defined on a different sets of variables. Defining $a_{i}=A\left(u^{i}\right), \ldots, g_{i}=$ $G\left(u^{i}\right)$ we have the equations

$$
\begin{aligned}
a_{1} y z+b_{1} y+c_{1} z & =e_{1} \\
a_{2} x z+b_{2} x+d_{2} z & =f_{2} ; \\
a_{3} x y+c_{3} x+d_{3} y & =g_{3} .
\end{aligned}
$$

By (13) and (15),

$$
y\left(a_{1} z+b_{1}\right)=e_{1}-c_{1} z \text { and } y\left(a_{3} x+d_{3}\right)=g_{3}-c_{3} x
$$

so that

$$
\left(e_{1}-c_{1} z\right)\left(a_{3} x+d_{3}\right)=\left(a_{1} z+b_{1}\right)\left(g_{3}-c_{3} x\right) .
$$

By $(14), z\left(a_{2} x+d_{2}\right)=f_{2}-b_{2} x$ and, by $(16)$,

$$
z\left(x\left(a_{3} c_{1}-a_{1} c_{3}\right)+a_{1} g_{3}+c_{1} d_{3}\right)=x\left(a_{3} e_{1}+b_{1} c_{3}\right)+d_{3} e_{1}-b_{1} g_{3} .
$$

We conclude that

$$
\left(x\left(a_{3} c_{1}-a_{1} c_{3}\right)+a_{1} g_{3}+c_{1} d_{3}\right)\left(f_{2}-b_{2} x\right)=\left(x\left(a_{3} e_{1}+b_{1} c_{3}\right)+d_{3} e_{1}-b_{1} g_{3}\right)\left(a_{2} x+d_{2}\right)
$$

so that, with

$$
\begin{aligned}
r & =a_{1} b_{2} c_{3}-a_{3} b_{2} c_{1}-a_{2} a_{3} e_{1}-a_{2} b_{1} c_{3}, \\
p & =a_{3} c_{1} f_{2}+a_{2} b_{1} g_{3}-a_{1} c_{3} f_{2}-a_{1} b_{2} g_{3}-b_{2} c_{1} d_{3}-a_{3} d_{2} e_{1}-b_{1} c_{3} d_{2}-a_{2} d_{3} e_{1}, \\
q & =a_{1} f_{2} g_{3}+c_{1} d_{3} f_{2}+b_{1} d_{2} g_{3}-d_{2} d_{3} e_{1}
\end{aligned}
$$

we have

$$
r x^{2}+p x+q=0
$$

Let $P, Q, R \in \mathbb{R}\left[\left(\theta_{\omega}^{\ell}\right)_{\omega \in \Omega, \ell \in\{1,2,3\}}\right]$ be the polynomials that correspond to $p, q, r$, that is,

$$
\begin{aligned}
R & =A_{1} B_{2} C_{3}-A_{3} B_{2} C_{1}-A_{2} A_{3} E_{1}-A_{2} B_{1} C_{3}, \\
P & =A_{3} C_{1} F_{2}+A_{2} B_{1} G_{3}-A_{1} C_{3} F_{2}-A_{1} B_{2} G_{3}-B_{2} C_{1} D_{3}-A_{3} D_{2} E_{1}-B_{1} C_{3} D_{2}-A_{2} D_{3} E_{1}, \\
Q & =A_{1} G_{3} F_{2}+C_{1} D_{3} F_{2}+B_{1} D_{2} G_{3}-D_{2} D_{3} E_{1} .
\end{aligned}
$$

We now define the set $\mathcal{F} \subseteq \mathbb{R}\left[\left(\theta_{\omega}^{\ell}\right)_{\omega \in \Omega, \ell \in\{1,2,3\}}\right]$ as follows: $\mathcal{F}$ consists of all polynomials of the form

$$
P, Q, R, \alpha_{i}, \alpha_{i} \beta_{j} \pm \alpha_{j} \beta_{i}, \alpha_{1} \beta_{2} \gamma_{3}+\alpha_{2} \beta_{3} \gamma_{1} \text { with } \alpha, \beta, \gamma \in\{A, \ldots, G\} \text { and } i, j \in\{1,2,3\}, i \neq j .
$$

Note that $\mathcal{F}$ is finite and it contains all polynomials that are explicitly used in the present proof.

We now assume that $u=\left(u^{1}, u^{2}, u^{3}\right) \in \mathcal{U}_{\mathcal{F}} \cap \mathcal{Y}$ so that there are infinitely many, hence at least three, values of $x$ that can be extended to a CMNE. By (18), $p=q=r=0$. Therefore $P, Q, R \in \mathcal{F}$ implies that $P=Q=R=0$. We distinguish the following cases:

Case 1: $A=0$. The system of equations (13), (14) and (15) is linear. The determinant of the associated matrix is $b_{1} c_{3} d_{2}+b_{2} c_{1} d_{3}$. There is more than one solution if and only if this determinant vanishes. As $B_{1} C_{3} D_{2}+B_{2} C_{1} D_{3} \in \mathcal{F}$, we conclude that $B_{1} C_{3} D_{2}+B_{2} C_{1} D_{3}=0$ so that, in particular, $B C D+B C D=$ $2 B C D=0$. As the cases $C=0$ and $D=0$ can be treated similarly, we only consider the case $B=0$. Then the system (13) - (15) becomes

$$
\begin{aligned}
c_{1} z & =e_{1} ; \\
d_{2} z & =f_{2} ; \\
c_{3} x+d_{3} y & =g_{3} .
\end{aligned}
$$


If $C=0$, then $E_{1} \in \mathcal{F}$ implies that $E_{1}=0$. As $A=0$, player 1 would be a dummy which was excluded. Similarly, if $D=0, F_{2} \in \mathcal{F}$ implies that $F_{2}=0$, i.e., player 2 would be a dummy which was also excluded. Hence, $C \neq 0 \neq D$. As $z>0$, the first two equations together with $C_{1}, E_{1}, D_{2}, F_{2}, E_{1} D_{2}-C_{1} F_{2} \in \mathcal{F}$ imply that $E_{1}, F_{2} \neq 0$ and $E_{1} D_{2}-C_{1} F_{2}=0$. From this, it follows that

$$
\frac{f_{2}}{d_{2}}=\frac{e_{1}}{c_{1}}=k
$$

and so $F=k D$ and $E=k C$. Thus, we have $z=k$ so that $z$ is uniquely determined. The polynomial $H$ becomes $H=C x z+D y z-E x-F y-G z+\theta_{222}=\theta_{222}-G z=\theta_{222}-G E / C$, and the distribution induced on outcomes is unique.

Case 2: $A \neq 0$. If $B=0$ or $C=0$, then $E=0$ as well because $R=0$ and $A_{2}, A_{3}, E_{1} \in \mathcal{F}$. By (13), the case $B=C=0$ cannot occur because $A_{1} \in \mathcal{F}$.

(1) We first consider the subcase $B=E=0$. As $z>0, y \neq 0$, and $A_{1} \in \mathcal{R}$, (13) implies that $a_{1} y+c_{1}=0$ so that $C \neq 0$ and $y$ is uniquely determined. As $P=0,\left(A_{3} C_{1}-A_{1} C_{3}\right) F_{2}=0$, and, as $Q=0,\left(A_{1} G_{3}+C_{1} D_{3}\right) F_{2}=0$. If $F=0$, then, by (14), $a_{2} x+d_{2}=0$ (because $z \neq 0$ ) so that $x$ is uniquely determined which was excluded. Hence, $A_{3} C_{1}=A_{1} C_{3}$ and $A_{1} G_{3}+C_{1} D_{3}=0$. As $C \neq 0$, there exists $k \in \mathbb{R} \backslash\{0\}$ such that $A=k C$. Moreover, we have that $D=-k G$.

Now, from (13), we have that $0=a_{1} y+c_{1}=(k y+1) c_{1}$. Since $c_{1} \neq 0$, we obtain $k y+1=0$. Using that $A=k C$, it follows that $A y=k y C=-C$. Substituting in (15), we obtain $G=y D$. The polynomial that determines the probabilities on outcomes becomes $H=A x y z+C x z+D y z-$ $F y-G z+\theta_{222}=-C x z+C x z+G z-F y-G z+\theta_{222}=\theta_{222}-F y$ and the distribution induced on outcomes is unique.

(2) The case $C=E=0$ may be treated analogously to the former case.

(3) $B C \neq 0$. We first claim that either $A=k B$ or $A=k C$, for some $k \in \mathbb{R}$. Since $R=0$, we have that $B_{2}\left(A_{1} C_{3}-A_{3} C_{1}\right)=A_{2}\left(A_{3} E_{1}+B_{1} C_{3}\right)$. If $A_{1} C_{3}-A_{3} C_{1}=0$ we conclude that $A=k C$ for some $k \in \mathbb{R}$, and the claim follows. Otherwise, we have that

$$
A_{2}=B_{2} \frac{a_{1} c_{3}-a_{3} c_{1}}{a_{3} e_{1}+b_{1} c_{3}}
$$

and it follows that $A=k B$ for some $k \in \mathbb{R}$. By our claim the following cases might occur:

(3.1) There exists $k \neq 0$ such that $A=k B$. Substituting this expression into $B_{2}\left(A_{1} C_{3}-A_{3} C_{1}\right)=$ $A_{2}\left(A_{3} E_{1}+B_{1} C_{3}\right)$ we obtain $C_{1}=-k E_{1}$. Hence, $C=-k E$. From (12) we may conclude that $k \in\{-1,-2\}$. Then,

$$
\begin{aligned}
& P=k\left(k F_{2}+D_{2}\right)\left(B_{1} E_{3}-B_{3} E_{1}\right), \\
& Q=\left(k F_{2}+D_{2}\right)\left(B_{1} G_{3}-D_{3} E_{1}\right),
\end{aligned}
$$

and the following subcases may occur:

i. $k F_{2}+D_{2}=0$ : Then $k F+D=0$. Equations (13) and (14) become

$$
\begin{aligned}
& b_{1} y(1+k z)=e_{1}(1+k z) ; \\
& b_{2} x(1+k z)=f_{2}(1+k z) .
\end{aligned}
$$

If $b_{2} x=f_{2}$, then $x$ is unique. Thus, from equation (14), we obtain that $1+k z=0$. It follows that $A z=k B z=-B$ and

$$
H=-B x y+B x y-k E x z-k F y z-E x-F y-G z+\theta_{222}=\theta_{222}-G z .
$$

ii. $k F_{2}+D_{2} \neq 0$ : Then $B_{1} E_{3}-B_{3} E_{1}=0$ and $B_{1} G_{3}-D_{3} E_{1}=0$. From $B \neq 0$ we obtain that $E=m B$. We consider the following subcases: 
A. $D=0$. Then, from $B_{1} G_{3}-D_{3} E_{1}=0$ and $B \neq 0$, we obtain that $G=0$. Equation (15) becomes $x\left(b_{3} y-e_{3}\right)=0$ and since $0<x<1$ we get $b_{3} y=e_{3}$, that is, $b_{3} y=m b_{3}$, i.e. $y=m$. Then, $H=m k B x z+m B x-m k B x z-m B x-m F+\theta_{222}=\theta_{222}-m F$.

B. $D \neq 0$. As $B_{1} G_{3}-D_{3} E_{1}=0$ there exists $\ell \in \mathbb{R}$ such that $E=\ell B$ and $G=\ell D$. Hence, $\ell=m$. Moreover, Equation (13) becomes $y(1+k z)=m(1+k z)$. If $1+k z \neq 0$, then $m=y$. Therefore,

$$
H=k m B x z+m B x-k m B x z+m D z-m B x-F m-m D z+\theta_{222}=\theta_{222}-F m
$$

so that the distribution is unique. If $1+k z=0$, then $k=-2$ because $k \in\{-1,-2\}$ and $0<z<1$. Hence, $z=1 / 2$ and (15) becomes $2 x b_{3}(m-y)=d_{3}(m-y)$. Now, $C=2 E \neq 0$ implies that $\omega:=\theta_{222}=\theta_{121} \neq \theta_{122}=\theta_{221}=: \omega^{\prime}$. We conclude that $B=E$ or $B=2 E$, i.e., $m=1$ or $m=1 / 2$, and claim that $B=E$ cannot occur. Assume, on the contrary, that $B=E$. Then $\omega^{\prime \prime}:=\theta_{112}=\theta_{212}$. As $m=1, D=G$ implies that $\theta_{211}=\theta_{212}=\omega^{\prime \prime}$. As $A=-2 B$, we conclude that

$$
A+B=\theta_{111}-\omega-\omega^{\prime \prime}+\omega=-B=\omega^{\prime}+\omega^{\prime \prime}-\omega^{\prime \prime}-\omega
$$

so that $\theta_{111}=\omega^{\prime \prime}$. Therefore $F=D$ and, hence (13) becomes $d_{2} / 2=d_{2}$. As $D \neq 0$ and $D \in \mathcal{F}$, the desired contradiction has been obtained.

If $B=2 E$, then $\theta_{112}=\omega$ and $\theta_{212}=\omega^{\prime}$. Moreover, $A=-2 B$ implies $\theta_{211}=\omega$ and $\theta_{111}=\omega^{\prime}$. Therefore we also have that $D=2 F$. Hence,

$$
H=-B x y+B x y+m B x+F y-m B x-F y-\frac{G}{2}+\theta_{222}=\theta_{222}-\frac{G}{2},
$$

and the distribution is unique.

(3.2) The case that $A=k C$ may be treated similarly.

\section{References}

GonzÁlez-Pimienta, C. (2010): "Generic finiteness of outcome distributions for two person game forms with three outcomes", Mathematical Social Sciences, 59, 364-365.

Govindan, S., and A. McLennan (1998): "Generic finiteness of outcome distributions for two person game forms with zero sum and common interest utilities", Mimeo, University of Western Ontario.

(2001): "On the generic finiteness of equilibrium outcome distributions in game forms", Econometrica, 69, 455-471.

Harsanyi, J. C. (1973): "Oddness of the number of equilibrium points: a new proof", International Journal of Game Theory, 2, 235-250.

Kreps, D. M., and R. Wilson (1982): "Sequential equilibria", Econometrica, 50, 863-894.

Kukushikin, N. S., C. Litan, and F. Marhuenda (2008): "On the generic finiteness of equilibrium outcome distributions in bimatrix game forms", Journal of Economic Theory, 139, 392-395.

Litan, C. M., and F. MARhuenda (2012): "Determinacy of equilibrium outcome distributions for zero sum and common utility games", Economics Letters, 115, 152 - 154.

Mas-Colell, A. (2010): "Generic finiteness of equilibrium payoffs for bimatrix games", Journal of Mathematical Economics, 46, 382-383.

PARK, I.-U. (1997): "Generic finiteness of equilibrium outcome distributions for sender-receiver cheaptalk games", Journal of Economic Theory, 76, 431-448.

Rosenmüller, J. (1971): "On a generalization of the Lemke-Howson algorithm to noncooperative nperson games", SIAM Journal on Applied Mathematics, 20, 73 - 79.

WiLson, R. (1971): "Computing equilibria of n-person games", SIAM Journal on Applied Mathematics, $20,80-87$. 